\title{
THE EFFECTS OF BACKORDER INFORMATION AND REDUCED-SETUP DISPATCHING UNDER REORDER POINT OR KANBAN REPLENISHMENT
}

\author{
S. T. Enns \\ Dept. of Mechanical and Manufacturing Engineering \\ University of Calgary \\ Calgary, AB., T2N-1N4, CANADA
}

\begin{abstract}
This research considers a single-stage, capacity-constrained workstation with circulating transporters used for replenishment and independent customer demand for completed parts. Characteristics of reorder point versus Kanban replenishment are examined. One objective is to determine the usefulness of backorder information for upstream order placement. A second objective is to investigate the benefits of dispatching based on setup time reduction. Performance is measured in terms inventory counts and the proportion of customer orders filled from stock. The area under tradeoff curves is used to evaluate comparisons statistically. Results show backorder information and setup time reduction both improve performance and that interaction effects are not significant. This indicates that reorder point systems can outperform Kanban systems whether or not dispatching based on setup time reduction is used.
\end{abstract}

\section{INTRODUCTION}

Continuous-review reorder point and Kanban replenishment systems are related in that both "pull" inventory downstream. The closeness of their relationship depends on how they are implemented or, in the case of research studies, the assumptions made in analysis or experimentation. There are many good references on reorder point and Kanban systems, including Sipper and Bulfin (1997), Silver et. al (1998) and Vollmann et. al (2005). Kanban modeling and research is reviewed by Groenevelt (1992).

Continuous-review reorder point studies commonly assume backorder information is incorporated in the order release decision. They also usually assume orders are instantaneously transmitted to upstream stations and that, given stock is available, shipments are immediately made. Shipping, or lead time, delays are then incurred prior to downstream delivery. The decision variables are commonly considered to be the reorder point and the order lot size.

Kanban studies do not assume the use of backorder information. The decision variables include the number of
Kanban cards and the Kanban lot size. If a two-card Kanban system is used, the number of both transportation and production cards needs to be determined. While two-card systems are common, especially for assembly operations, further consideration in this study is given only to singlecard systems.

Another type of continuous-review replenishment that is even simpler than a Kanban system is the two-bin system. In this system the reorder point is based on the size of the smaller bin and the order lot is equal to the larger bin size. No backorder information is used and only one lot size order can be in the replenishment loop at a time.

The consideration of transportation issues further complicates the design and analysis of replenishment systems. In reorder point systems upstream supply stations are not likely to make shipments instantaneously when an order is received since there is likely to be a delay before a transporter becomes available. In Kanban systems it is also possible that orders are transmitted instantaneously to upstream supply stations using an "electronic" Kanban, where lights or other signals replace the use of physical cards. This makes order placement similar to a reorder point system with electronic order placement. Delays in both cases will be dependent on the number of transporters or frequency of shipment.

More often Kanban cards circulate and act to deliver orders to upstream supply stations in the form of cards or containers. Therefore order placements are not instantaneous and this may affect transportation variable settings. Furthermore, if inventory is not available upstream there may still will be a shipping delay and the length of this delay will depend on whether the transporter circulates continuously or waits upstream until the order can be filled. This illustrates that assumptions about order placement and shipment delays are not always clear cut for either reorder point or Kanban systems and must be clearly stated.

With capacity-constrained workstations there are also issues of priority dispatching for lots in queue. There has been a fair amount of discussion on setup time reduction in Kanban systems under circumstances where two or more 
lots of the same part type may be in queue along with other part type lots. Priority rules have been developed that facilitate processing multiple lots of the same part type sequentially, thus reducing the number of setups. Although there has been less discussion of setup time reduction for reorder point systems, the same approach can be used.

Comparisons of replenishment systems must be very clear on the assumptions made. As well, they must attempt to identify the characteristics of the systems which actually determine differences in performance it they are to be fully understood.

Few studies have directly compared Kanban and reorder point strategies. Krajewski et al. (1987) conducted comparisons to determine important planning and control factors having an impact on manufacturing performance. Results of Kanban and reorder point comparisons showed that there was not much difference in performance. Setup time reduction, lot size reduction, defect reduction and other factors, which can be applied using any replenishment strategy, were found to be more significant than the choice of replenishment logic.

Yang (1998) compared results using Kanban and reorder point strategies in a single-stage, capacity-constrained system. He concluded that results using the Kanban strategy were superior. However, the Kanban strategy was modified to essentially allow lot-sizes of one and dispatching which facilitated setup time reduction. Since the lot sizing and dispatching policies were not consistently applied, it is difficult to attribute the improved performance to the Kanban logic in this study.

Suwanruji and Enns (2006a) concluded performance using reorder points dominated that using Kanbans in a distribution system without capacity constraints. Suwanruji and Enns (2006b) further concluded that while reorder points are generally better under time-varying demand for either capacity constrained or unconstrained networks of stations, there are circumstances with level demand patterns and capacity constraints where a Kanban system may slightly outperform a reorder point system. The conclusions of both studies were based on instantaneous order transmission and shipment assumptions (i.e. infinite number of transporters).

This study is an extension to another study examining the differences between continuous-review reorder point and single-card Kanban systems under level and seasonal demand patterns (Enns, 2006). The previous results, using the proportion of customer orders filled from stock as a performance measure, lead to the conclusion reorder point systems outperform Kanban systems. This was mainly due to instantaneous order transmission for the reorder point system, which resulted in better response to changes in demand. However backorder information also proved to be value when average backorders were examined, especially under seasonal demand. In this follow-up study the value of backorder information is examined more closely under conditions where order transmission assumptions are consistent across all replenishment systems. A second factor relates to priority dispatching and the effect of rules based on setup reduction. As well, analysis of possible interaction effects is introduced.

In the next section the logic used in modeling reorder point systems is compared with that used in Kanban systems. Following this, the simulation model used in this research is presented. The experimental design and results are described in later sections.

\section{REPLENISHMENT LOGIC AND EXECUTION}

The continuous-review reorder point strategy assumes that orders are triggered as soon as inventory falls below an order point. Individual order points must be defined for each part type $i$, or stock keeping unit (sku). If it is assumed that order quantities are multiples of some lot size $Q^{*}$, the following equation can be used to describe the order quantity, $Q$, at some given time $t$.

$Q_{i, t}=\max \left(0,\left\lceil\frac{O P_{i}-\left(I n v_{i, t}+O R_{i, t}+O T_{i, t}+O Q_{i, t}-B O_{i, t}\right)}{Q_{i}^{*}}\right\rceil Q_{i}^{*}\right)$

where:

$O P_{i} \quad$ - Order point for sku $i$

$I n v_{i, t} \quad$ - Quantity of sku $i$ finished goods in stock

$O R_{i, t} \quad$ - Quantity of sku $i$ orders released to supplier but not yet filled

$O T_{i, t} \quad$ - Quantity of sku $i$ in transit

$O Q_{i, t} \quad$ - Quantity of sku $i$ in queue or on machine

$B O_{i, t} \quad$ - Quantity of sku $i$ backordered

Under the assumption that processed parts become available to meet customer demand, Inv represents finished goods in stock. With ROP strategies it is often assumed that orders are placed instantaneously to upstream stations. In this case $O R$ represents orders that have not been filled due to lack of inventory at upstream stations or orders waiting upstream for a transporter. An upstream station could refer to either an external supplier or an internal station performing a preceding operation.

With a single-card Kanban strategy the total number of cards in circulation for each stock keeping unit (sku) must be specified. If it is assumed the Kanban card is released upstream for recirulation when a container is completely depleted and $K_{T O T, i}$ is the total number of cards in the replenishment loop, the order quantity, $Q$, at time $t$ is given by the following equation.

$Q_{i, t}=\max \left(0,\left\lceil\frac{\left(K_{T O T, i}-1\right) Q_{i}^{*}-\left(I n v_{i, t}+O R_{i, t}+O T_{i, t}+O Q_{i, t}\right)+1}{Q_{i}^{*}}\right\rceil Q_{i}^{*}\right)$

In this case the interpretation of variables $I n v, O T$ and $O Q$ is the same as for the reorder point strategy in Equation (1). However Kanban orders often travel back to the supplier as 
a Kanban card, or container, with the transporter. In this case the $O R$ quantity could include the quantity represented by cards waiting downstream for pickup and cards in transport to the supplier. If the supplier does not have sufficient inventory, the orders that have not been filled due to lack of upstream inventory or orders waiting upstream for a transporter would also be included, as in the case with the reorder point strategy.

In comparing the logic used in reorder point and Kanban strategies, a key difference is in the consideration of backorders. Since Kanban systems do not consider backorders, they lend themselves to simpler implementations. There is no requirement to keep track of anything beyond the status at certain Kanban card locations. However, one could argue that the additional backorder information used by reorder point systems should be beneficial, especially under uncertain or time-varying demand.

In a single-card strategy the following equation provides a breakdown of where Kanban cards may be located and where time delays may occur for sku $i$. Reference to Figure 1 can provide clarification.

$$
K_{T O T, i}=K_{S, i}+K_{I, i}+K_{Q, i}+K_{P, i}+K_{W, i}+K_{E, i}+K_{O, i}
$$

where:

$K_{T O T, i} \quad$ - Cards in total system

$K_{S, i} \quad$ - Cards at upstream (supplier) station

$K_{I, i} \quad$ - Cards on inbound transporter containers

$K_{Q, i} \quad$ - Cards on containers in queue

$K_{P, i} \quad$ - Cards on container being processed

$K_{W, i} \quad$ - Cards on finished goods containers

$K_{E, i} \quad$ - Cards waiting for transporter pickup

$K_{O, i} \quad$ - Cards outbound to supplier

If it is assumed that the supplier always has inventory available and that Kanban cards circulate with transporters that are constantly cycling, then $K_{S}$ will be zero. However, it is also possible to signal orders to the upstream station instantaneously rather than using Kanban cards to communicate the order. In this modified Kanban strategy transporters may still circulate containers from the downstream station but the quantity picked up at the upstream station depends on the orders received in the time interval since the last transporter departed. Since orders would typically need to wait for a transporter to make a downstream pickup, $K_{S}$ would not be zero even if the upstream station always has stock. However under these instantaneous ordering assumptions, $K_{E}$ and $K_{O}$ are always zero.

\section{A SINGLE-STAGE REPLENISHMENT SCENARIO}

A simple production scenario was modeled using Arena 5.0 discrete-event simulation software (Kelton, et al, 2004). This scenario is shown in Figure 1.
Two part types, or stock keeping units (skus), are supplied from different upstream stations. These upstream stations are assumed to always have sufficient stock to fill orders. Lot size quantities of each sku are shipped from the upstream station and arrive at a capacity-constrained workstation. If the workstation machine is busy when a lot arrives, it must join a queue. When a lot is loaded on the machine, the parts in the lot are processed on a part-by-part basis. Both part types are assumed to have identical lot sizes, setup times and part processing times. The lot sizes are 5, the setup times are 0.015 and the part processing times are 0.006 time units. There is no uncertainty associated with the setup or processing times.

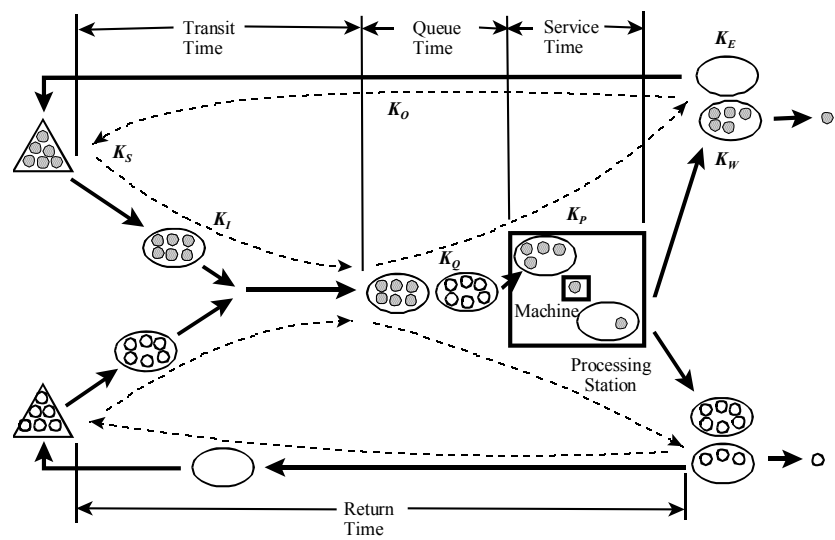

Figure 1: Configuration of Single-Stage System

Once the lot is complete, it is immediately available to meet customer demand as finished goods inventory. Customer arrivals for each type of sku are independent and can be described as a Poisson distribution. Each customer requires only one unit. However the demand pattern is seasonal so the demand rate changes over time. The seasonality for both skus is described by a sinusoidal pattern with a mean demand rate of 50, amplitude of 10 and cycle length of 250 time units. The demand pattern for the two skus are offset by 125 time units. As a result the aggregate workload requirements at the processing station are fairly constant over time even though the demand for each of the skus is seasonal. The actual demand rate is determined once every time unit based on the expected demand from the sinusoidal demand pattern multiplied by an uncertainty factor. This uncertainty factor is sampled from a Normal distribution with a mean of 1.0 and a standard deviation of 0.10 .

There are 8 transporters in each of the two replenishment loops. These transports circulate continuously and are spaced so the interarrival times at the machine are constant. The time to move from the upstream supplier to the processing station is 0.8 time units. The time to move from the processing station to the upstream supplier is also 0.8 time units. The transporters are not assumed to have any other delays so the circulation time is 1.6 time units. It 
is assumed the transporters can carry no lots, a single lot or multiple lots of parts.

The replenishment orders are assumed to be transmitted instantaneously to the upstream station when either the reorder point is reached or a Kanban container of finished goods is empty. The next transporter to circulate past the upstream station then picks up the number of lots ordered and ships them to the processing workstation. In other words, $K_{E}$ and $K_{O}$ in Equation (3) are equal to 0. The only real difference between the reorder point and Kanban systems, as implemented in this research, is that the reorder point system uses backorder information while the Kanban system does not.

\section{SIMULATION EXPERIMENTAL DESIGN}

The experimental design consisted of two factors, each with two levels. The first factor, BO, indicated no use (-1 level) or use (+1 level) of backorder information. It will be noted that the -1 level describes the Kanban system and the +1 level describes the reorder point system.

The second factor, PDR, indicated the type of priority dispatching rule used for lots in queue at the capacityconstrained workstation. The first dispatch rule was a simple first-come-first-serve (FCFS) rule (-1 level). A setup time is incurred every time a new lot is processed, even if the lot is of the same part type. The second priority dispatch rule considered both requirements and setup time reduction (+1 level).

The setup reduction rule $(\mathrm{PDR}=+1)$ was implemented as follows. If only one type of part was available for processing, a lot of this part type was loaded. A setup time was incurred only if the previous lot was of a different type. If both part types were in queue, the minimum finished goods inventory required to avoid stockout was calculated for each part type, assuming a lot of the other part was being produced first. This benchmark inventory level was based on average demand rates. In other words, the average demand over the time interval required to produce both part types, with setups, was used to determine a minimum finished goods inventory benchmark. If both part types had inventory above the benchmark, the same part type as was previously processed was loaded again so that a setup could be avoided. If both part types were below the benchmark so stockout of at least one part type was likely, the same part type as was previously processed was loaded again to minimize setups. If one part type was above the benchmark and the other below, the part type below the benchmark was loaded next, whether or not a setup would be incurred.

Performance was measured in terms of the total inventory counts in the system and the proportion of customer demand filled from stock. If a customer arrived during a stockout, the desired item was backordered and then deliv- ered immediately upon replenishment of finished goods inventory.

There is a tradeoff between minimizing inventory levels and achieving good delivery performance. Since the objective is to determine the relative dominance of one replenishment strategy over another across different service levels, it is necessary to generate tradeoff curves for both types of measures. This was done across all combinations of experimental factor settings by running each combination of factor settings at various inventory levels. Inventory levels were adjusted by changing the order point in increments of 5 and by changing the number of Kanban cards in increments of 1 . Typically 10 settings were used for each full-factorial combination of settings to generate a tradeoff curve. In other words, 40 combinations of factor settings and tradeoff curve settings were required to generate one full set of tradeoff curves.

Three replications were run at each of the 40 combinations of settings. Common random numbers were used as a variance reduction technique. A warmup period of 250 time units was used to reach steady-state conditions and data was then collected over the next 2500 time units. If a time unit is considered to be one day and there are 250 working days per year, this is the equivalent of 5 years of data collection per replication.

\section{EXPERIMENTAL RESULTS}

The experimental results are summarized in figures 2 and 3 . Figure 2 shows the average inventory as a function of delivery performance when no backorder information is used $(\mathrm{BO}=-1)$. Figure 3 shows similar results with backorder information $(\mathrm{BO}=+1)$. These curves are based on averaging the results across the three replications at each combination of experimental settings. Comparing figures 2 and 3 indicates that backorder information improves performance slightly. Lower curves indicate that less inventory is required to obtain any given level of delivery performance. It is also obvious from both figures 2 and 3 that the dispatch rule reducing setups $(\mathrm{PDR}=+1)$ is preferable to the FCFS dispatch rule (PDR=-1), which incurs a setup for each lot.

The machine utilization, defined to include both the machine setup and part processing times, for $\mathrm{PDR}=-1$ was $90 \%$. With $\mathrm{PDR}=+1$ the utilization ranged from about $73 \%$ when the proportion delivered from stock was 0.50 , to $67 \%$ when the proportion delivered from stock was 1.00 . At higher delivery service levels more inventory was required and the longer average queue lengths ahead of the machine resulted in more opportunities to eliminate lot setups. Fewer setups resulted in lower utilization. 


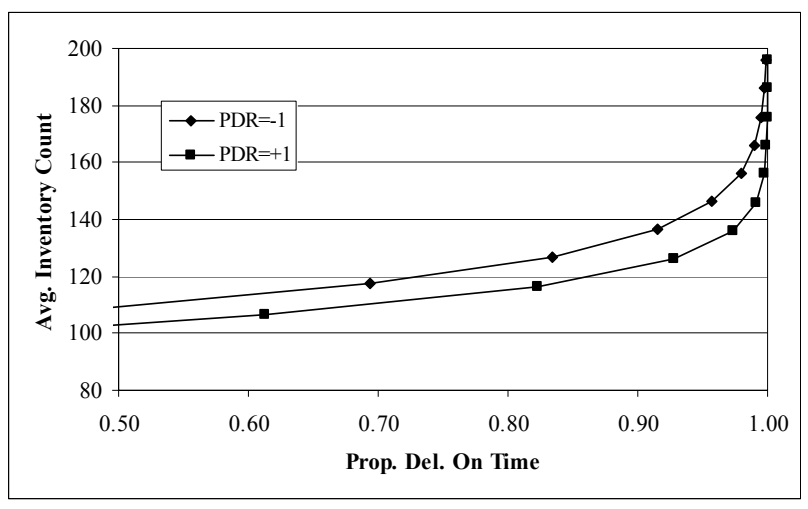

Figure 2: Tradeoff curves with $\mathrm{BO}=-1$

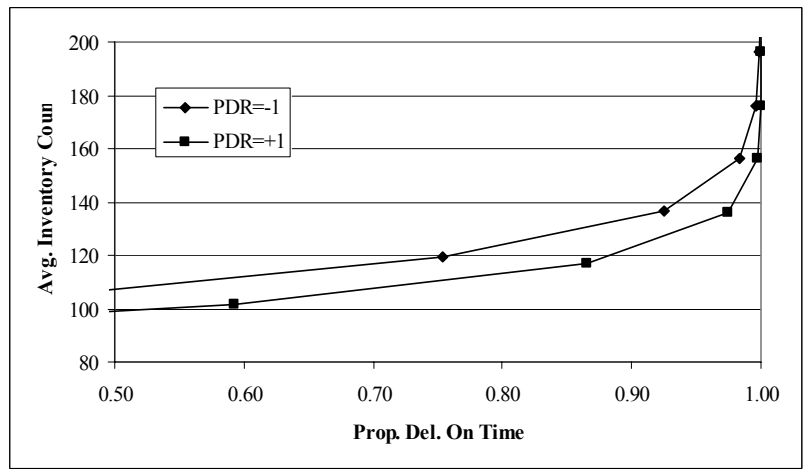

Figure 3: Tradeoff Curves with $\mathrm{BO}=+1$

The results were also analyzed to determine statistical significance and identify possible interaction effects. The area under each of the 12 curves generated (2 BO levels* 2 PDR levels * 3 replications) was calculated using a lower service bound of 0.50 and an upper service bound of 1.00 . A lower area indicates better performance. These areas were then treated as the response in an analysis of variance (ANOVA) model. The results are shown in Table 1. The coefficient of determination $\left(R^{2}\right)$ was $99.45 \%$ and residual analysis indicated that ANOVA modeling assumptions were not being violated.

Table 1: ANOVA Results

$\begin{array}{lrrrrrr}\text { Source } & \text { DF } & \text { Seq SS } & \text { Adj SS } & \text { Adj MS } & \text { F } & \text { P } \\ \text { PDR } & 1 & 73.949 & 73.949 & 73.949 & 1420.66 & 0.000 \\ \text { BO } & 1 & 1.560 & 1.560 & 1.560 & 29.97 & 0.001 \\ \text { PDR*BO } & 1 & 0.270 & 0.270 & 0.270 & 5.18 & 0.052 \\ \text { Error } & 8 & 0.416 & 0.416 & 0.052 & & \\ \text { Total } & 11 & 76.195 & & & & \end{array}$

The ANOVA results show that both the priority dispatch rule (PDR) factor and backorder information (BO) factor were statistically significant, although backorder information provided relatively little improvement. The in- teraction effect between the PDR and BO factors was not significant at the $95 \%$ confidence level.

Figure 4 shows a main effects plot for the results while figure 5 shows an interaction plot. These graphs confirm that the dispatching rule based on setup reduction and the use of backorder information both result in improved performance.

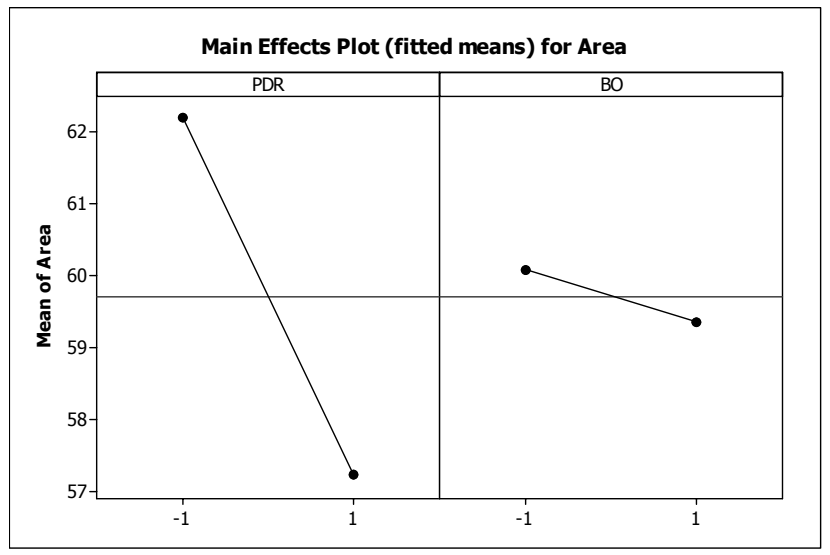

Figure 4: Main Effects Plot

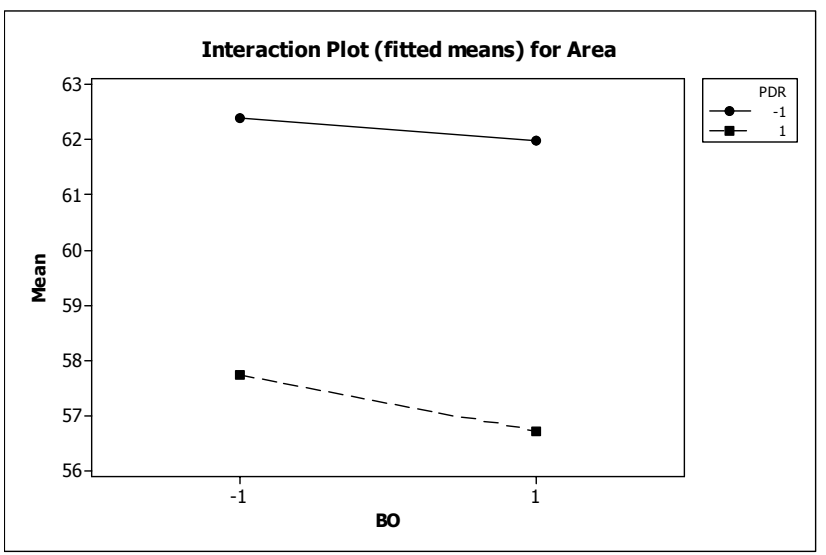

Figure 5: Interaction Plot

\section{CONCLUSIONS}

This study has identified some of the issues concerning the comparison of "pull" type replenishment systems. The use of backorder information and dispatching based on setup reduction were specifically examined. Both were found to be beneficial through use of both tradeoff curve and ANOVA comparison techniques. The advantage of using ANOVA based on measuring the area under tradeoff curves was demonstrated. This technique allows differences in performance to be statistically verified when there are tradeoffs between two performance measures and it is desirable to determine dominance across a range of delivery service levels. As well, interaction effects between various factors can be determined. 


\section{ACKNOWLEDGMENTS}

This research was supported in part by a grant from the National Science and Engineering Research Council (NSERC) of Canada.

\section{REFERENCES}

Enns, S.T. 2006. A comparison of kanban and reorder point replenishment at capacity-constrained workstation. In Proceedings of Summer Computer Simulation Conference, forthcoming.

Groenevelt, Harry. 1992. "The Just-In-Time System." in Handbooks in Operations Research and Management Science, Vol. 4, Logistics of Production and Inventory, Edited by S.C. Graves, A.H.G Rinnooy Kan and P.H. Zipkin, Elsevier Science Publishers, Amsterdam, The Netherlands.

Kelton, W.D., R.P. Sadowski, and D.T. Sturrock. 2004. Simulation with Arena, $3^{\text {rd }}$ Ed., New York, NY: McGraw-Hill.

Krajewski, L.J., B.E. King, L.P. Ritzman, and D.S. Wong. 1987. Kanban, MRP, and shaping the manufacturing environment." Management Science, Vol. 33, No. 1, 39-57.

Silver, E.A., D.F. Pyke, and R. Peterson. 1998. Inventory Management and Production Planning and Scheduling, $3^{\text {rd }}$ Ed., New York, NY: John Wiley \& Sons.

Sipper, D., and R.L. Bulfin. 1997. Production: Planning, Control and Integration, New York, NY: McGrawHill.
Suwanruji, P., and S.T. Enns. 2001. The role of information, logic and control in comparing approaches to supply chain management. in Proceedings of the $1^{\text {st }}$ International Conference on Integrated Logistics, ed. K.T. Yeo and S. Pokharel, 93-100.

Suwanruji, P., and S.T. Enns. 2006a. Comparison of supply chain replenishment strategies in a non-capacitated distribution system. International Journal of Risk Assessment and Management, forthcoming.

Suwanruji, P., and S.T. Enns. 2006b. Evaluating the effects of capacity constraints and demand patterns on supply chain replenishment strategies. International Journal of Production Research, forthcoming.

Vollmann, T.E., W.L. Berry, D.C. Whybark, and F.R. Jacobs. 2005. Manufacturing Planning and Control for Supply Chain Management, $5^{\text {th }}$ Ed., New York, NY: McGraw-Hill.

Yang, K.K. 1998. A comparison of reorder point and kanban policies for a single machine production system. Production Planning and Control, Vol. 9, No. 4, 385390.

\section{AUTHOR BIOGRAPHIES}

SILVANUS T. ENNS is an Associate Professor at the University of Calgary. He received a $\mathrm{PhD}$ from the University of Minnesota. His research interests lie in the development of algorithms to support enhanced MRP performance as well as various aspects of job shop, batch production and supply chain modeling and analysis. His email address is <enns@ucalgary . ca>. 